\title{
Acht Elemente einer grundlegenden Reform des Steuer- und Transfersystems
}

\begin{abstract}
Vor dem Hintergrund höherer Überschüsse als in den öffentlichen Haushalten in Deutschland eingeplant, wird derzeit über Steuersenkungen diskutiert. Eigentlich sollten kurzfristige und ungeplante Schwankungen in den öffentlichen Haushalten kein Anlass sein, Ausgaben oder Steuern anzupassen. Konjunkturpolitisch wären Steuersenkungen bei staatlichen Überschüssen und Steuererhöhungen bei Defiziten sogar unerwünscht, weil eine derartige Steuerpolitik prozyklisch wirken, Konjunkturschwankungen also verstärken würde. Ausgaben- und Steuerpolitiken sollten letztlich langfristig orientiert sein. Allerdings weisen die öffentlichen Haushalte in Deutschland seit längerer Zeit Überschüsse auf. Ist es also an der Zeit, dauerhaft die Steuern zu senken?
\end{abstract}

\section{Sind Steuern und Staatsausgaben in Deutschland zu hoch oder zu niedrig?}

Kritiker von Steuersenkungen führen häufig ins Feld, es gebe viele unerledigte öffentliche Aufgaben, für die eher höhere als niedrigere Steuereinnahmen gebraucht werden. Befürworter von Steuersenkungen setzen dem entgegen, die Staatsausgaben seien in den letzten Jahren bereits erhöht worden, oft mit zweifelhaftem Nutzen,

(C) Der/die Autor(en) 2020. Open Access: Dieser Artikel wird unter der Creative Commons Namensnennung 4.0 International Lizenz (http:// creativecommons.org/licenses/by/4.0/deed.de) veröffentlicht.

Open Access wird durch die ZBW - Leibniz-Informationszentrum Wirtschaft gefördert.

Prof. Dr. Dr. h. c. Clemens Fuest ist Präsident des ifo Instituts und Inhaber des Lehrstuhls für Nationalökonomie und Finanzwissenschaft an der LudwigMaximilians-Universität München.

Prof. Dr. Andreas Peichl ist Leiter des ifo Zentrums für Makroökonomik und Befragungen und Professor für Volkswirtschaftslehre, insbesondere Makroökonomie und Finanzwissenschaft, an der Ludwig-Maximilians-Universität München.
Steuersenkungen seien notwendig, um verschwenderische Staatsausgaben einzudämmen.

Letztlich verdeutlicht diese Debatte, dass unterschiedliche Gruppen der Bevölkerung divergierende Präferenzen und wirtschaftliche Interessen haben, was die Höhe der Staatsausgaben angeht. Wer selbst nur ein geringes Einkommen hat und deshalb keine Einkommensteuern zahlt, aber von öffentlichen Leistungen profitiert, wird von Einkommensteuersenkungen und damit geringeren öffentlichen Leistungen weniger begeistert sein als jemand, der ein hohes Einkommen hat und steuerlich entsprechend viel beitragen muss. Unabhängig von der eigenen Steuerlast messen einige Bürger öffentlichen Leistungen einen hohen Wert zu, andere wünschen sich eher niedrige Steuern, um den privaten Konsum auszudehnen. In welche Richtung sich die Steuer- und Transferpolitik bewegt, ist immer auch eine Frage von normativen bzw. politischen Werturteilen und abhängig von Kompromissen im politischen Prozess. Im Kontext der Umverteilungspolitik lässt sich im internationalen Vergleich festhalten, dass Deutschland sich im Mittelfeld bewegt. Dies gilt auch für die Entwicklung sowohl von Einkommensungleichheit als auch der mittleren Einkommen der Haushalte, wo Deutschland im internationalen Vergleich relativ gut da steht.

Insofern gibt es verteilungspolitisch keinen akuten Handlungsbedarf. Gleichwohl ist es jenseits der oben aufgeführten Interessenkonflikte interessant zu fragen, wie sich der Anteil des Staats an der gesamtwirtschaftlichen Leistung in den letzten Jahren entwickelt hat. Bei einem steigenden Anteil von Steuereinnahmen und öffentlichen Ausgaben an der gesamtwirtschaftlichen Leistung ist die Argumentation für Steuersenkungen überzeugender als in einer Situation, in der Steuer- und Ausgabenquoten für längere Zeit gefallen sind.

Abbildung 1 illustriert die öffentlichen Primärausgaben (Gesamtausgaben abzüglich der Ausgaben für Zinsen), mit denen ja keine öffentlichen Leistungen finanziert werden. Als Ausgangspunkt wird das Jahr 2012 gewählt, das erste Jahr, in dem Deutschland gesamtstaatlich ausgeglichene öffentliche Haushalte aufwies. Es zeigt sich, dass

1 Vgl. A. Peichl, N. Pestel, S. Siegloch: Ist Deutschland wirklich so progressiv? Einkommensumverteilung im europäischen Vergleich, in: DIW Vierteljahrshefte zur Wirtschaftsforschung, 82. Jg. (2013), H. 1, S. 111-127; sowie P. Hufe, A. Peichl: Inequality and Unfairness in Europe, in: CESifo Forum, 19. Jg. (2018), H. 2, S. 26-34. 
Abbildung 1

Öffentliche Primärausgaben in Deutschland

in \% des Bruttoinlandsprodukts

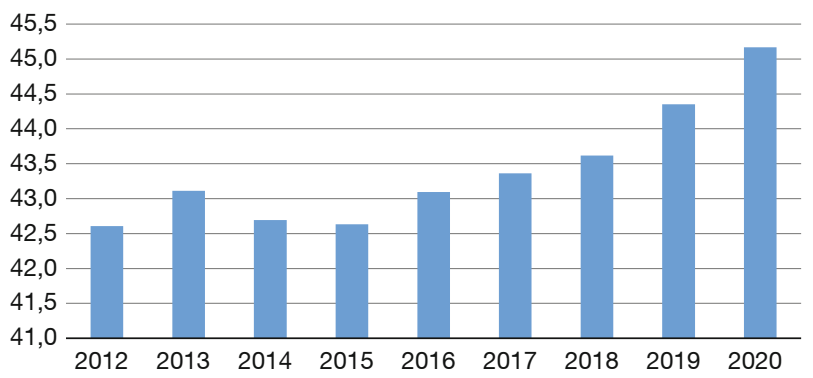

Quelle: Statistisches Bundesamt; eigene Berechnungen.

\section{Abbildung 2}

Steuern und Sozialabgaben in Deutschland

in \% des Bruttoinlandsprodukts

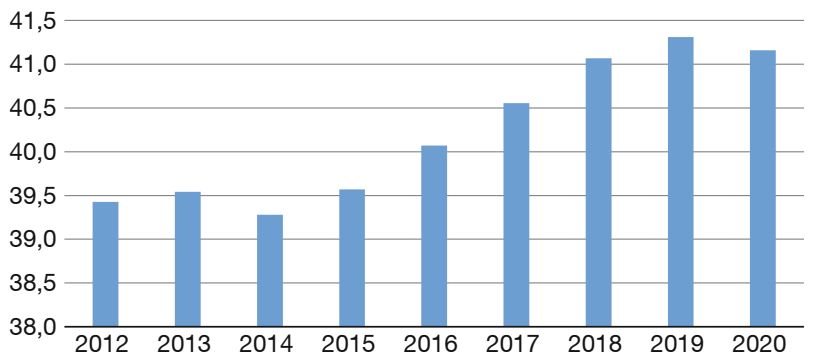

Quelle: Statistisches Bundesamt; eigene Berechnungen.

\section{Abbildung 3}

Persönliche Einkommensteuern und indirekte Steuern

in \% des Gesamtsteueraufkommens

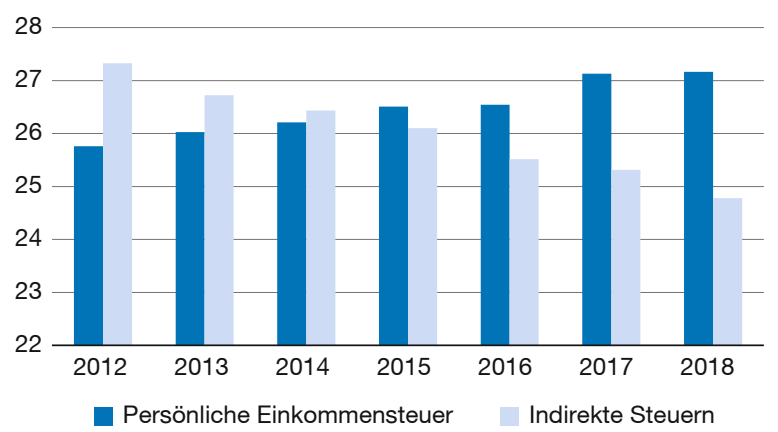

Quelle: Statistisches Bundesamt.

die öffentlichen Ausgaben im Verhältnis zur gesamtwirtschaftlichen Leistung im betrachteten Zeitraum um 2,6 Prozentpunkte gestiegen sind.

Abbildung 2 illustriert die Entwicklung der Steuern und Sozialabgaben im gleichen Zeitraum. Auch die Abgaben- quote hat deutlich, um 1,8 Prozentpunkte, zugenommen. Es zeigt sich, dass der öffentliche Sektor seit 2012 erheblich gewachsen ist. Diese Verschiebung zwischen öffentlicher und privater Verwendung von Ressourcen kann sehr unterschiedlich bewertet werden. Beispielsweise kann man argumentieren, dass bei alternder Bevölkerung staatliche Ausgaben für Gesundheit und Pflege zwangsläufig zunehmen. Allerdings muss man im Zeitraum von 2012 bis 2020 in Rechnung stellen, dass die Beschäftigung sich in Deutschland sehr gut entwickelt hat und angesichts sinkenden Bedarfs an staatlicher Unterstützung für Arbeitslose eigentlich insgesamt sinkende Staatsausgaben zu erwarten wären. Angesichts der Entwicklung der öffentlichen Ausgaben in den letzten Jahren erscheint die These, die Staatsausgaben sollten weiter ausgedehnt werden, weniger überzeugend als es bei sinkender Staatsausgabenquote der Fall wäre.

\section{Welche Steuern senken?}

Wenn man sich nun dafür entscheidet, Steuern zu senken, welche Steuern sollten es sein? Auch hier haben unterschiedliche Bevölkerungsgruppen sehr unterschiedliche Interessen. Zur Orientierung ist es interessant, die Entwicklung des Beitrags der wichtigsten Steuern zum Gesamtsteueraufkommen in den Jahren seit 2012 zu betrachten. Es zeigt sich, dass es eine Verschiebung weg von indirekten Steuern hin zu Einkommensteuern gegeben hat (vgl. Abbildung 3). Das ist vor allem durch zwei Faktoren getrieben. Zum einen führt die kalte Progression zu einem stetigen Anstieg der Einkommensteuerlast. Hier hat es zwischenzeitlich immer wieder Ausgleichsmaßnehmen gegeben, sie waren jedoch zu gering, um die Wirkung der kalten Progression zu neutralisieren. Zum anderen ist der Anteil der Erwerbstätigen an der Bevölkerung in Deutschland seit 2012 gewachsen. ${ }^{2}$ Das führt zu einem wachsenden Anteil der Einkommensteuern am Steueraufkommen insgesamt, ohne dass die Steuerlast pro Steuerzahler notwendigerweise zunimmt.

Neben Senkungen der Einkommensteuer oder der Mehrwertsteuer werden immer wieder Entlastungen bei den Unternehmensteuern diskutiert. Abbildung 4 zeigt, dass der Beitrag der Steuern auf Unternehmensgewinne zum Gesamtsteueraufkommen seit 2012 ebenfalls gestiegen ist. Das Aufkommen der Unternehmensgewinnsteuern hängt allerdings stärker vom Konjunkturzyklus ab als das Aufkommen aus Einkommensteuer oder Mehrwertsteuer. Angesichts der zumindest bis 2018 sehr positiven Konjunkturentwicklung ist es nicht überraschend, dass die Gewinnsteuern schneller steigen als das Gesamtsteueraufkommen.

2 Im Jahr 2012 lag die Erwerbsquote in Deutschland bei 54,9\%, im Jahr 2018 bei $55,7 \%$. 


\section{Abbildung 4}

\section{Steuern auf Unternehmensgewinne}

in \% des Gesamtsteueraufkommens

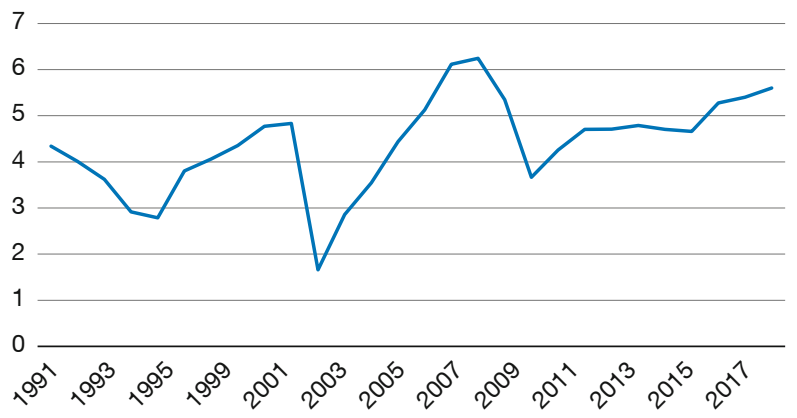

Quelle: Statistisches Bundesamt; eigene Berechnungen.

Entlastungen bei den Unternehmensteuern werden auch weniger wegen Verschiebungen in der Steueraufkommensstruktur diskutiert, sondern in erster Linie wegen des internationalen Steuerwettbewerbs. In den letzten Jahren haben viele wichtige Industrieländer die Unternehmensteuern deutlich gesenkt, zuletzt die USA mit der großen Steuerreform, die 2018 in Kraft trat. ${ }^{3}$ Eine Senkung der Unternehmensbesteuerung würde vor allem darauf abzielen, die Attraktivität Deutschlands als Standort für Investitionen und Arbeitsplätze zu steigern und Anreize zu mindern, Gewinne in Niedrigsteuerländer zu verlagern.

\section{Acht Elemente für eine grundlegende Reform des} Steuer- und Transfersystems

Wenn es bei den anstehenden Änderungen nur darum geht, die Steuerzahler auf pragmatische Weise zu entlasten, könnte man sich darauf beschränken, die Einkommensteuer durch eine Tarifanpassung zu senken. Derzeit wird auch diskutiert, die Abschaffung des Solidaritätszuschlags für die "unteren $90 \%$ " um sechs Monate vorzuziehen. In diesem Fall würde es sich um eine einmalige Entlastung handeln.

Damit würde die Finanzpolitik jedoch die Chance verpassen. Größere Steuerreformen sind aus politökonomischen Gründen leichter durchzuführen, wenn sie mit einer steuerlichen Entlastung kombiniert werden. Bei aufkommensneutralen Reformen gibt es dagegen stets Verlierer, die sich wehren. Deshalb spricht viel dafür, die vorhandenen finanziellen Spielräume für eine umfassendere Reform des Steuer- und Transfersystems zu nutzen.

3 Vgl. Wissenschaftlicher Beirat beim Bundesministerium der Finanzen: US-Steuerreform 2018 - Steuerpolitische Folgerungen für Deutschland, Stellungnahme, Nr. 1, 2019; und D. Boumans, C. Fuest, C. Krolage, K. Wohlrabe: Expected Effects of the US Tax Reform on Other Countries: Global and Local Survey Evidence, CESifo Working Paper, Nr. 7491, 2019.
Eine solche Reform sollte aus unserer Sicht die folgenden Punkte beinhalten: ${ }^{4}$

1. Die vollständige Abschaffung des Solidaritätszuschlags für alle Steuerzahler, ${ }^{5}$ kombiniert mit einer grundlegenden Reform der Einkommensteuer, die einen neuen Einkommensteuertarif, eine Reform der Familienbesteuerung (Realsplitting statt Ehegattensplitting), ${ }^{6}$ einen Abbau von ungerechtfertigten Steuersubventionen sowie einen „Tarif auf Rädern“ für einen automatischen Ausgleich der kalten Progression beinhalten sollte; ${ }^{7}$

2. Unternehmensbesteuerung: Abschaffung der Gewerbesteuer, Körperschaftsteuersatz von $25 \%$, Restriktionen der Verlustverrechnung abbauen; 8

3. Reform der Kommunalbesteuerung (z.B. VierSäulen-Modell der Reformkommission Stiftung Marktwirtschaft); 9

4. Reform der Erbschaftsteuer: Abbau von Vergünstigungen für Betriebsvermögen und selbst genutzte Immobilien, unter Beibehaltung hoher Freibeträge, im Gegenzug ein einheitlicher, niedriger Steuersatz (z. B. $10 \%$ );

5. einfache Grundsteuer: statt der unnötig komplizierten wertbasierten Grundsteuer eine rein flächenbasierte Steuer oder eine Kombination aus Bodenrichtwerten und Gebäudeflächen; ${ }^{10}$

4 Wir beschränken uns hier aus Platzgründen auf Reformen im Steuerund Transfersystem im engeren Sinne und gehen nicht auf das System der Sozialversicherungen und Sozialversicherungsbeiträge ein, das ebenfalls reformbedürftig ist.

5 Vgl. M. Blömer, L. Dörr, C. Fuest, M. Mosler, A. Peichl, N. Potrafke: Was bei einer Reform des Solidaritätszuschlags zu beachten ist, in: ifo Schnelldienst, 72. Jg. (2019), H. 16.

6 Vgl. Wissenschaftlicher Beirat beim Bundesministerium der Finanzen: Zur Reform der Besteuerung von Ehegatten, Gutachten, Nr. 2/ 2018.

7 Vergleiche hierzu auch M. Löffler, A. Peichl, N. Pestel, H. Schneider, S. Siegloch: Effizient, einfach und gerecht: Ein integriertes System zur Reform von Einkommensteuer und Sozialabgaben, in: Perspektiven der Wirtschaftspolitik, Verein für Sozialpolitik, 13. Jg. (2012), H. 3, S. 196-213.

8 Eine Abschaffung der Gewerbesteuer ist nicht nur aus Effizienzgründen, sondern auch verteilungspolitisch sinnvoll, da diese Steuer besonders stark auf die Löhne der Beschäftigten überwälzt wird. Vgl. C. Fuest, A. Peichl, S. Siegloch: Wer trägt die Lasten von Steuern auf Unternehmensgewinne? - Lehren aus den Erfahrungen mit der deutschen Gewerbesteuer, in: ifo Schnelldienst, 70. Jg. (2017), H. 24, S. 22-27.

9 Vgl. Stiftung Marktwirtschaft: Kommission Steuergesetzbuch: Bericht der Arbeitsgruppe Kommunalfinanzen, Juli 2006, https://www.stiftungmarktwirtschaft.de/fileadmin/user_upload/Steuern/Bericht_der_AG_ Kommunalfinanzen_Kommission_Steuergesetzbuch.pdf (10.3.2020).

10 Vgl. C. Fuest, L. Immel, V. Meier, F. Neumeier: (2018), Reformoptionen für die Grundsteuer B: Eine empirische Analyse der Belastungswirkungen, in: ifo Schnelldienst, 71. Jg. (2018), H. 22, S. 23-29; und Wissenschaftlicher Beirat beim Bundesministerium der Finanzen: Die Reform der Grundsteuer: Ein Kompromissvorschlag, Stellungnahme, Nr. 2, 2019 
6. Umsatzsteuer: Bereich der ermäßigten Steuersätze überprüfen und deutlich einschränken;

7. Niedrigeinkommensbereich: Transfers und Anrechnungsregeln so anpassen, dass die derzeit bestehende Teilzeitfalle mit impliziter Grenzsteuerbelastung von teilweise über $100 \%$ beseitigt wird;11

8. keine erneute Einführung einer Nettovermögensteuer: wenn man alle Einkommen und Erbschaften richtig be-

11 Vgl. M. Blömer, C. Fuest, A. Peichl: Die Hartz IV-Reformdebatte, in: ifo Schnelldienst, 72. Jg. (2019), H. 6, S. 21-25; dies.: Was sind die wichtigsten Ansatzpunkte für eine Reform von Hartz IV?, in: Wirtschaftsdienst, 99. Jg. (2019), H. 4, S. 243-247, https://www.wirtschaftsdienst.eu/inhalt/jahr/2019/heft/4/beitrag/hartz-iv-reformeiner-umstrittenen-politischen-massnahme.html (10.3.2020); sowie dies.: Raus aus der Niedrigeinkommensfalle(!), Der ifo-Vorschlag zur Reform des Grundsicherungssystems, in: ifo Schnelldienst, 72. Jg. (2019), H. 4, S. 34-43. steuert, dann bedarf es keiner weiteren Vermögensbesteuerung, die sehr hohe Erhebungskosten und Ausweichreaktionen mit sich bringen würde.

In der finanzpolitischen Debatte werden häufig mehr oder weniger willkürlich ausgewählte Einzelmaßnahmen vorgeschlagen, um die Steuerzahler zu entlasten oder bestimmte Staatsausgaben auszudehnen. Das ist verständlich, weil Kompromisse bei umfassenderen Reformen schwieriger und aufwändiger zu finden sind. Trotzdem wäre das in der aktuellen Lage unbefriedigend. Die steuerpolitische Debatte sollte breiter geführt werden. Die vorhandenen finanziellen Spielräume eröffnen die Möglichkeit einer umfassenderen Reform des deutschen Steuersystems. Eine solche Reform könnte sowohl die Akzeptanz und Fairness des Steuersystems steigern als auch Impulse für mehr Investitionen und Beschäftigung setzen. Diese Chance ungenutzt zu lassen wäre leichtfertig. 